\title{
A note on $k$-colorability of $P_{5}$-free graphs
}

\author{
Chính T. Hoàng* Marcin Kamiński ${ }^{\dagger} \quad$ Vadim Lozin $^{\ddagger} \quad$ J. Sawada ${ }^{\S} \quad$ X. Shu ${ }^{\Uparrow}$
}

\begin{abstract}
A polynomial time algorithm that determines whether or not, for a fixed $k$, a $P_{5}$-free graph can be $k$-colored is presented in this paper. If such a coloring exists, the algorithm will produce a valid $k$-coloring.
\end{abstract}

Keywords: $P_{5}$-free, graph coloring, dominating clique

\section{Introduction}

Graph coloring is among the most important and applicable graph problems. The $k$-colorability problem is the question of whether or not the vertices of a graph can be colored with one of $k$ colors so that no two adjacent vertices are assigned the same color. In general, the $k$-colorability problem is NPcomplete [10]. Even for planar graphs with no vertex degree exceeding 4, the problem is NP-complete [5]. However, for other classes of graphs, like perfect graphs [8], the problem is polynomial-time solvable. For the following special class of perfect graphs, there are efficient polynomial time algorithms for finding optimal colorings: chordal graphs [6], weakly chordal graphs [9], and comparability graphs [4]. For more information on perfect graphs, see [1], [3], and [7].

Another interesting class of graphs are those that are $P_{t}$-free, that is, graphs with no chordless paths $v_{1}, v_{2}, \ldots, v_{t}$ of length $t-1$ as induced subgraph for some fixed $t$. If $t=3$ or $t=4$, then there exists efficient algorithms to answer the $k$-colorability question (see [3]). However, it is known that CHROMATIC NUMBER for $P_{5}$-free graphs is NP-complete [11]. Thus, it is of some interest to consider the problem of $k$-coloring a $P_{t}$-free graph for some fixed $k \geq 3$ and $t \geq 5$. Taking this parameterization

${ }^{*}$ Physics and Computer Science, Wilfrid Laurier University, Canada. Research supported by NSERC. E-mail: choang@wlu. ca

${ }^{\dagger}$ RUTCOR, Rutgers University, 640 Bartholomew Road, Piscataway, NJ 08854, USA. E-mail: mkaminski@rutcor.rutgers.edu

${ }^{\ddagger}$ Mathematics Institute, University of Warwick, Coventry CV4 7AL UK. E-mail : V. Lozin@warwick.ac.uk

${ }^{\S}$ Computing and Information Science, University of Guelph, Canada. Research supported by NSERC. E-mail: sawada@cis.uoguelph.ca

${ }^{\top}$ Computing and Information Science, University of Guelph, Canada. E-mail : xshu@uoguelph. ca 


\begin{tabular}{|c|c|c|c|c|c|c|c|c|c|}
\hline$k \backslash t$ & 3 & 4 & 5 & 6 & 7 & 8 & $\ldots$ & 12 & $\ldots$ \\
\hline 3 & $O(m)$ & $O(m)$ & $O\left(n^{\alpha}\right)$ & $O\left(m n^{\alpha}\right)$ & $?$ & $?$ & $?$ & $?$ & $\ldots$ \\
4 & $O(m)$ & $O(m)$ & $\mathbf{? ?}$ & $?$ & $?$ & $?$ & $?$ & $N P_{c}$ & $\ldots$ \\
5 & $O(m)$ & $O(m)$ & $\mathbf{? ?}$ & $?$ & $?$ & $N P_{c}$ & $N P_{c}$ & $N P_{c}$ & $\ldots$ \\
6 & $O(m)$ & $O(m)$ & $\mathbf{? ?}$ & $?$ & $?$ & $N P_{c}$ & $N P_{c}$ & $N P_{c}$ & $\ldots$ \\
7 & $O(m)$ & $O(m)$ & $\mathbf{? ?}$ & $?$ & $?$ & $N P_{c}$ & $N P_{c}$ & $N P_{c}$ & $\ldots$ \\
$\ldots$ & $\ldots$ & $\ldots$ & $\ldots$ & $\ldots$ & $\ldots$ & $\ldots$ & $\ldots$ & $\ldots$ & $\ldots$ \\
\hline
\end{tabular}

Table 1: Known complexities for $k$-colorability of $P_{t}$-free graphs

into account, a snapshot of the known complexities for the $k$-colorability problem of $P_{t}$-free graphs is given in Table 1. From this chart we can see that there is a polynomial algorithm for the 3-colorability of $P_{6}$-free graphs [12].

In this paper we focus on $P_{5}$-free graphs. Notice that when $k=3$, the colorability question for $P_{5^{-}}$ free graphs can be answered in polynomial time (see [13]). We obtain a theorem (Theorem 2) on the structure of $P_{5}$-free graphs and use it to design a polynomial-time algorithm that determines whether a $P_{5}$-free graph can be $k$-colored. If such a coloring exists, then the algorithm will yield a valid $k$ coloring.

The remainder of the paper is presented as follows. In Section 2 we present relevant definitions, concepts, and notations. Then in Section 3, we present our recursive polynomial-time algorithm that answers the $k$-colorability question for $P_{5}$-free graphs.

\section{Background and Definitions}

In this section we provide the necessary background and definitions used in the rest of the paper. For starters, we assume that $G=(V, E)$ is a simple undirected graph where $|V|=n$ and $|E|=m$. If $A$ is a subset of $V$, then we let $G(A)$ denote the subgraph of $G$ induced by $A$.

DEFINITION 1 A set of vertices $A$ is said to dominate another set $B$, if every vertex in $B$ is adjacent to at least one vertex in $A$.

The following structural result about $P_{5}$-free graphs is from Bacsó and Tuza [2]:

THEOREM 1 Every connected $P_{5}$-free graph has either a dominating clique or a dominating $P_{3}$.

DEFINITION 2 Given a graph $G$, an integer $k$ and for each vertex $v$, a list $l(v)$ of $k$ colors, the $k$-list coloring problem asks whether or not there is a coloring of the vertices of $G$ such that each vertex receives a color from its list. 
DEFINITION 3 The restricted $k$-list coloring problem is the $k$-list coloring problem in which the lists $l(v)$ of colors are subsets of $\{1,2, \ldots, k\}$.

Our general approach is to take an instance of a specific coloring problem $\Phi$ for a given graph and replace it with a polynomial number of instances $\phi_{1}, \phi_{2}, \phi_{3}, \ldots$ such that the answer to $\Phi$ is "yes" if and only if there is some instance $\phi_{k}$ that also answers "yes".

For example, consider a graph with a dominating vertex $u$ where each vertex has color list $\{1,2,3,4,5\}$. This listing corresponds to our initial instance $\Phi$. Now, by considering different ways to color $u$, the following set of four instances will be equivalent to $\Phi$ :

$\phi_{1}: l(u)=\{1\}$ and the remaining vertices have color lists $\{2,3,4,5\}$,

$\phi_{2}: l(u)=\{2\}$ and the remaining vertices have color lists $\{1,3,4,5\}$,

$\phi_{3}: l(u)=\{3\}$ and the remaining vertices have color lists $\{1,2,4,5\}$,

$\phi_{4}: l(u)=\{4,5\}$ and the remaining vertices have color lists $\{1,2,3,4,5\}$.

In general, if we recursively apply such an approach we would end up with an exponential number of equivalent coloring instances to $\Phi$.

\section{The Algorithm}

Let $G$ be a connected $P_{5}$-free graph. This section describes a polynomial time algorithm that decides whether or not $G$ is $k$-colorable. The algorithm is outlined in 3 steps. Step 2 requires some extra structural analysis and is presented in more detail in the following subsection.

1. Identify and color a maximal dominating clique or a $P_{3}$ if no such clique exists (Theorem 1 ). This partitions the vertices into fixed sets indexed by available colors. For example, if a $P_{5}$-free graph has a dominating $K_{3}$ (and no dominating $K_{4}$ ) colored with $\{1,2,3\}$ and $k=4$, then the fixed sets would be given by: $S_{124}, S_{134}, S_{234}, S_{14}, S_{24}, S_{34}$. For an illustration, see Figure 1 . Note that all the vertices in $S_{124}$ are adjacent to the vertex colored 3 and thus have color lists $\{1,2,4\}$. This gives rise to our original restricted list-coloring instance $\Phi$. Although the illustration in Figure 1 does not show it, it is possible for there to be edges between any two fixed sets.

2. Two vertices are dependent if there is an edge between them and the intersection of their color lists is non-empty. In this step, we remove all dependencies between each pair of fixed sets. This process, detailed in the following subsection, will create a polynomial number of coloring instances $\left\{\phi_{1}, \phi_{2}, \phi_{3}, \ldots\right\}$ equivalent to $\Phi$.

3. For each instance $\phi_{i}$ from Step 2 the dependencies between each pair of fixed sets has been removed which means that the vertices within each fixed set can be colored independently. Thus, 


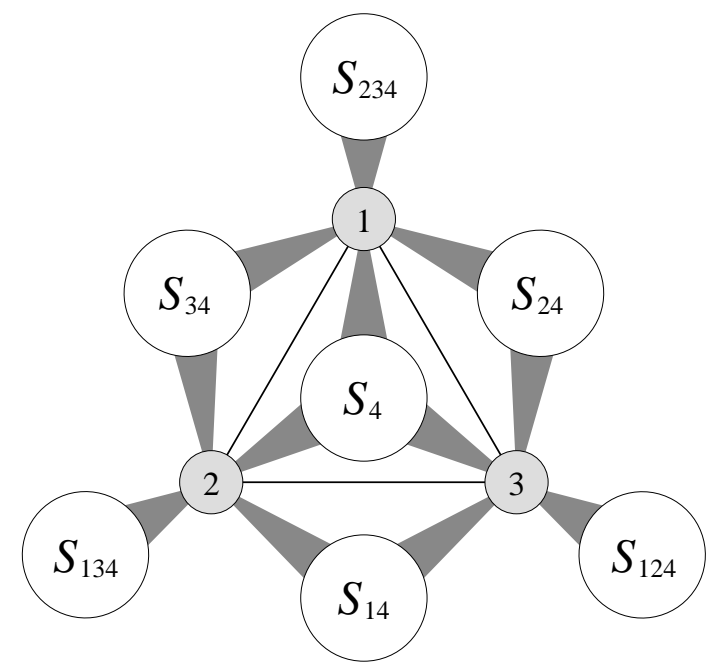

Figure 1: The fixed sets in a $P_{5}$-free graph with a dominating $K_{3}$ where $k=4$.

for each instance $\phi_{i}$ we recursively see if each fixed set can be colored with the corresponding restricted color lists (the base case is when the color lists are a single color). If one such instance provides a valid $k$-coloring then return the coloring. Otherwise, the graph is not $k$-colorable.

As mentioned, the difficult part is reducing the dependencies between each pair of fixed sets (Step 2).

\subsection{Removing the Dependencies Between Two Fixed Sets}

Let $S_{\text {list }}$ denote a fixed set of vertices with color list given by list. We partition each such fixed set into dynamic sets that each represent a unique subset of the colors in list. For example: $S_{123}=$ $P_{123} \cup P_{12} \cup P_{13} \cup P_{23} \cup P_{1} \cup P_{2} \cup P_{3}$. Initially, $S_{123}=P_{123}$ and the remaining sets in the partition are empty. However, as we start removing dependencies, these sets will dynamically change. For example, if a vertex $u$ is initially in $P_{123}$ and one of its neighbors gets colored 2, then $u$ will be removed from $P_{123}$ and added to $P_{13}$.

Recall that our goal is to remove the dependencies between two fixed sets $S_{p}$ and $S_{q}$. To do this, we remove the dependencies between each pair $(P, Q)$ where $P$ is a dynamic subset of $S_{p}$ and $Q$ is a dynamic subset of $S_{q}$. Let $\operatorname{col}(P)$ and $\operatorname{col}(Q)$ denote the color lists for the vertices in $P$ and $Q$ respectively. By visiting these pairs in order from largest to smallest with respect to $|\operatorname{col}(P)|$ and then $|\operatorname{col}(Q)|$, we ensure that we only need to consider each pair once. Applying this approach, the crux of the reduction process is to remove the dependencies between a pair $(P, Q)$ by creating at most a polynomial number of equivalent colorings.

Now, observe that there exists a vertex $v$ from the dominating set found in Step 1 of the algorithm that dominates every vertex in one set, but is not adjacent to any vertex in the other. This is because $P$ and $Q$ are subsets of different fixed sets. WLOG assume that $v$ dominates $Q$. 


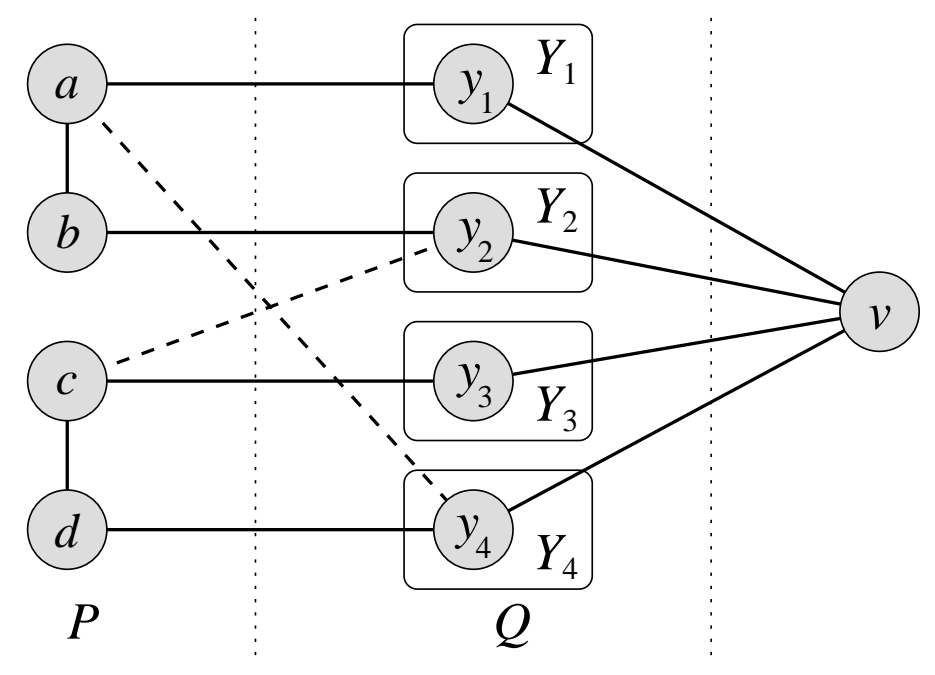

Figure 2: Illustration for proof of Theorem 2

THEOREM 2 Let $H$ be a $P_{5}$-free graph partitioned into three sets $P, Q$ and $\{v\}$ where $v$ is adjacent to every vertex in $Q$ but not adjacent to any vertex in $P$. If we let $Q^{\prime}$ denote all components of $H(Q)$ that are adjacent to some vertex in $P$ then one of the following must hold.

1. There exists exactly one special component $C$ in $G(P)$ that contains two vertices a and $b$ such that a is adjacent to some component $Y_{1} \in G(Q)$ but not adjacent to another component $Y_{2} \in G(Q)$ while $b$ is adjacent to $Y_{2}$ but not $Y_{1}$.

2. There is a vertex $x$ that dominates every component in $Q^{\prime}$, except at most one (call it $T$ ).

Proof: Suppose that there are two unique components $X_{1}, X_{2} \in G(P)$ with $a, b \in X_{1}$ and $c, d \in X_{2}$ and components $Y_{1} \neq Y_{2}$ and $Y_{3} \neq Y_{4}$ from $G(Q)$ such that:

- $a$ is adjacent to $Y_{1}$ but not adjacent to $Y_{2}$,

- $b$ is adjacent to $Y_{2}$ but not adjacent to $Y_{1}$,

- $c$ is adjacent to $Y_{3}$ but not adjacent to $Y_{4}$,

- $d$ is adjacent to $Y_{4}$ but not adjacent to $Y_{3}$.

Let $y_{1}$ (respectively, $y_{2}, y_{3}, y_{4}$ ) be a vertex in $Y_{1}$ (respectively, $Y_{2}, Y_{3}, Y_{4}$ ) that is adjacent to $a$ (respectively, $b, c, d)$ and not $b$ (respectively, $a, d, c)$. Since $H$ is $P_{5}$-free, there must be edges $(a, b)$ and $(c, d)$, otherwise $a, y_{1}, v, y_{2}, b$ or $c, y_{3}, v, y_{4}, d$ would be $P_{5}$ s. An illustration of these vertices and components is given in Figure 2.

Suppose $Y_{2}=Y_{3}$. Then $b$ is not adjacent to $y_{3}$, for otherwise there exists a $P_{5} a, b, y_{3}, c, d$. Now, there exists a $P_{5} y_{1}, a, b, y_{2}, y_{3}$ (if $y_{2}$ is adjacent to $y_{3}$ ) or a $P_{5} a, b, y_{2}, v, y_{3}$ (if $y_{2}$ is not adjacent to $y_{3}$ ). Thus, $Y_{2}$ and $Y_{3}$ must be unique components. Similarly, we have $Y_{2} \neq Y_{4}$. Now since $b, y_{2}, v, y_{3}, c$ cannot be 


\author{
Procedure RemoveDependencies $\left(P^{\prime}, Q, \varphi\right)$ \\ if no dependencies between $P^{\prime}$ and $Q$ \\ then output $\varphi$ \\ else find $x, T$ from Theorem 2 \\ for each $c \in \operatorname{col}(P) \cap \operatorname{col}(Q)$ do \\ output ReduceComponent $(T, \varphi$ with $x$ colored $c)$ \\ RemoveDependencies $\left(P^{\prime}-\{x\}, Q, \varphi\right.$ with $\left.l(x)=\operatorname{col}(P)-\operatorname{col}(C)\right)$
}

Figure 3: Algorithm to remove dependencies between two dynamic sets $P^{\prime}$ and $Q$ (with no special component $C$ ) by creating an equivalent set of coloring instances with the dependences removed.

a $P_{5}$, either $b$ is adjacent to $y_{3}$ or $c$ must be adjacent to $y_{2}$. WLOG, suppose the latter. Now $a, b, y_{2}, v, y_{4}$ implies that either $a$ or $b$ is adjacent to $y_{4}$. If $y_{4}$ is adjacent to $b$ but not $a$, then $a, b, y_{4}, d, c$ would be a $P_{5}$ which implies that $a$ must be adjacent to $y_{4}$ anyway. Thus, we end up with a $P_{5} a, y_{4}, v, y_{2}, c$ which is a contradiction to the graph being $P_{5}$-free. Thus there must be at most one special component $C$.

Now suppose that there is no special component $C$. Let $Q^{\prime}$ denote all components in $Q$ that are adjacent to some vertex in $P$. Let $x$ be a vertex in $P$ that is adjacent to the largest number of components in $Q^{\prime}$. Suppose that $x$ is not adjacent to a component $T$ of $Q^{\prime}$. Thus there is some other vertex $x^{\prime} \in P$ adjacent to $T$. The maximality of $x$ implies there is a component $S$ of $Q$ such that $x$ is adjacent to $S$ but $x^{\prime}$ is not. If $x$ is not adjacent to $x^{\prime}$, then there is a $P_{5}$ with $x, s, v, r, x^{\prime}$ with some vertices $s \in S, r \in T$. Thus $x$ and $x^{\prime}$ belongs to a special component $C$ of $P$ - a contradiction. Thus, $x$ must be adjacent to all components of $Q^{\prime}$.

If there are two components $A, B$ of $Q^{\prime}$ that are not dominated by $x$, then there are adjacent vertices $a, b \in A$, adjacent vertices $c, d \in B$ such that $x$ is adjacent to $a, c$ but not to $b, d$; but now the five vertices $b, a, x, c, d$ form a $P_{5}$.

Given a list-coloring instance $\phi$ of a $P_{5}$-free graph, we will at some points need to reduce the color lists for a given connected component $C$. This can be done by considering all possible ways to color $C$ 's dominating clique or $P_{3}$ (Theorem 1). Since there are a constant number of vertices in such a dominating set, we obtain a constant number of new instances that together are equivalent to $\phi$. For future reference, we call this function that returns this set of equivalent instances ReduceComponent $(C, \phi)$. If $C$ is empty, the function simply returns $\phi$.

Using this procedure along with Theorem 2, we can remove the dependencies between two dynamic sets $P$ and $Q$ for a given list-coloring instance $\phi$. First, we find the special component $C$ if it exists, and set $C=\emptyset$ otherwise. Then we call ReduceComponent $(C, \phi)$ which will effectively remove all vertices in $C$ from $P$ as their color lists change. Then, for each resulting coloring instance $\varphi$ we remove the remaining dependencies between $P^{\prime}=P-C$ and $Q$ by applying procedure RemoveDepencencies $\left(P^{\prime}, Q, \varphi\right)$ shown in Figure 3.1. In this procedure we find a vertex $x$ and component $T$ from Theorem 2, since we know that the special component $C$ has already been handled. If $T$ does not exist, then we set $T=\emptyset$. Now by considering each color in $\operatorname{col}\left(P^{\prime}\right) \cap \operatorname{col}(Q)$ along with the list $\operatorname{col}\left(P^{\prime}\right)-\operatorname{col}(Q)$ we can create a set of equivalent instances to $\varphi$ (as described in Section 2). If we 
modify $\varphi$ by assigning $x$ a color from $\operatorname{col}\left(P^{\prime}\right) \cap \operatorname{col}(Q)$, then all vertices in $Q$ adjacent to $x$ will have their color list reduced by the color of $x$. Thus, only the vertices in $T$ may still have dependencies with the original set $P$ - but these dependencies can be removed by a single call to ReduceComponent. In the single remaining instance where we modify $\varphi$ by assigning $x$ the color list $\operatorname{col}(P)-\operatorname{col}(Q)$, we simply repeat this process (at most $|P|$ times) by setting $P^{\prime}=P^{\prime}-\{x\}$ until there are no remaining dependencies between $P$ and $Q$. Thus, each iteration of RemoveDependencies produces a constant number of instances with no dependencies between $P$ and $Q$ and one instance in which the size of $P^{\prime}$ is reduced by one at least one.

The output of this step is $O(n)$ list-coloring instances (that are obtained in polynomial time), with no dependencies between $P$ and $Q$, that together are equivalent to the original instance $\phi$. Since there are a constant number of pairs of dynamic sets for each pair of fixed sets, and since there are a constant number of pairs of fixed sets, this proves the following theorem:

THEOREM 3 Determining whether or not a $P_{5}$-free graph can be colored with $k$-colors can be decided in polynomial time.

\section{Summary}

In this paper, we obtain a theorem (Theorem 2) on the structure of $P_{5}$-free graphs and use it to design a polynomial-time algorithm that determines whether a $P_{5}$-free graph can be $k$-colored. The algorithm recursively uses list coloring techniques and thus its complexity is high even though it is polynomial, as is the case with all list coloring algorithms. In a related paper (in preparation), we will give a slightly faster algorithm also based on list coloring techniques, however this algorithm provides less insight into the structure of $P_{5}$-free graphs. It would be of interest to find a polynomial-time algorithm to $k$-color a $P_{5}$-free graph without using list coloring techniques.

Continuing with this vein of research, the following open problems are perhaps the next interesting avenues for future research:

- Does there exist a polynomial time algorithm to determine whether or not a $P_{7}$-free graph can 3-colored?

- Does there exist a polynomial time algorithm to determine whether or not a $P_{6}$-free graph can 4-colored?

- Is the problem of $k$-coloring a $P_{7}$-free graph NP-complete for any $k \geq 3$ ?

Two other related open problems are to determine the complexities of the MAXIMUM INDEPENDENT SET and MINIMUM INDEPENDENT DOMINATING SET problems on $P_{5}$-free graphs. 


\section{References}

[1] Jorge L. Ramirez Alfonsin, Bruce A. Reed, Perfect Graphs, John Wiley \& Sons, LTD, 2001

[2] G. Bacsó and Z. Tuza, Dominating cliques in $P_{5}$-free graphs, Period. Math. Hungar. Vol. 21 No. 4 (1990) 303-308.

[3] C. Berge and V. Chvátal (eds.), Topics on perfect graphs, North-Holland, Amsterdam, 1984.

[4] S. Even, A. Pnueli and A. Lempel, Permutation graphs and transitive graphs, J. Assoc. Comput. Mach. 19 (1972) 400-410.

[5] M. R. Garey, D. S. Johnson and L. Stockmeyer, Some simplified NP-complete problems, Theo. Comput. Sci. 1, (1976) 237-267.

[6] F. Gavril, Algorithms for minimum coloring, maximum clique, minimum coloring by cliques, and maximum independent set of a chordal graph, SIAM J. Comput. 1, (1972) 180-187.

[7] M. C. Golumbic, Algorithmic graph theory and perfect graphs, Academic Press, New York, 1980.

[8] M. Grötschel, L. Lovász and A. Schrjver, The ellipsoid method and its consequences in combinatorial optimization, Combinatorica 1, (1981) 169-197.

[9] R. Hayward, C. T. Hoàng and F. Maffray, Optimizing weakly triangulated graphs, Graphs and Combinatorics 5 (1989) 339-349.

[10] R. M. Karp, Reducibility among combinatorial problems. In: R. E. Miller and J. W. Thatcher (eds), Complexity of Computer Computations, Plenum Press, New York, (1972) 85-103.

[11] D. Kral, J. Kratochvil, Z. Tuza and G. J. Woeginger, Complexity of coloring graphs without forbidden induced subgraphs, in: WG 2001, LNCS 2204, (2001) 254-262.

[12] B. Randerath and I. Schiermeyer, 3-Colorability $\in P$ for $P_{6}$-free graphs, Discrete Applied Mathematics, Vol. 136 No. 2-3 (2004) 299-313.

[13] J. Sgall, G.J. Woeginger, The complexity of coloring graphs without long induced paths, Acta Cybernet. Vol. 15 No. 1 (2001) 107-117. 